\title{
Habilidades blandas en estudiantes de ingeniería, un estudio comparativo 2020
}

\section{Soft skills in engineering students, a comparative study 2020}

\author{
Rodolfo Rojas Gallo ${ }^{1}$ \\ ${ }^{1}$ Facultad de Ingeniería Económica, Estadística y Ciencias Sociales, Universidad Nacional de Ingeniería, \\ Lima, Perú \\ *E-mail: rrojas@uni.edu.pe \\ Recibido (Received): 23/06/2020 Aceptado (Accepted): 07/10/2020 Publicado (Published): 15/12/2020
}

\begin{abstract}
RESUMEN
La presente investigación denominada Habilidades blandas en estudiantes de ingeniería, un estudio comparativo 2020 tuvo como objetivo describir, medir y comparar el nivel de habilidades blandas en estudiantes de Ingeniería en dos universidades públicas de Lima, una denominada A y otra denominada B. El estudio fue de tipo cuantitativo, transversal, de diseño no experimental con una muestra de 244 estudiantes, entre las dos universidades. Se aplicó un cuestionario de 22 ítems preguntas, validado por Claudet (2018), vía on line. Los resultados fueron: que los estudiantes de la Universidad A y los de la Universidad B presentan habilidades blandas en Asertividad en un $55 \%$ en nivel aceptable, en Liderazgo $45 \%$ nivel bueno y en Conciencia y actualidad, un $45 \%$ medianamente aceptable. Presentan diferencias no muy notorias en las habilidades en general, y en las dimensiones entre,$+-5 \%$. Siendo las de menor desarrollo, dominio del idioma inglés, comunicación fluida y los trabajos con proyectos.
\end{abstract}

Palabras Clave: Habilidades blandas, competencias, estudiantes de ingeniería, asertividad, liderazgo conciencia.

\begin{abstract}
The present investigation, called Soft Skills in Engineering Students, a comparative study, 2020, aimed to describe, measure, and compare the level of soft skills in engineering students from two public universities in Lima, one named $A$ and the other named $B$. The study was of a crosssectional quantitative type of non-experimental design with a sample of 244 students from each university. A 22-question questionnaire was applied, validated by Claudet (2018), via online. His main results were that his students before University A have a relatively higher level in Assertiveness, Leadership and Communal Culture. This corroborates the working hypothesis.
\end{abstract}

Keywords: Soft skills, Assertiveness, leadership, social awareness

\section{PROBLEMÁTICA}

Varias investigaciones y artículos científicos consultados, dan cuenta de que las universidades de en varios países no preparan a sus estudiantes para su incursión en el mundo laboral. Llegan con toda la información académica que les han entregado en los años de pregrado, pero sin mucha experiencia y habilidades blandas (Sarmiento,2019). En nuestro país, las empresas requieren profesionales con experticias diferentes a los conocimientos, 
como la creatividad, liderazgo, comunicación fluida, don de mando y capacidad de superar problemas y emprender nuevos retos. (Ponce, 2018). También manifiestan que, actualmente, no solo es necesario el conocimiento de las ciencias, como matemática, física, química, o de las tecnologías. Para el éxito profesional, es imprescindible el desenvolvimiento personal con características distinguibles; urge, entonces, aplicar las llamadas "soft skills". Las habilidades blandas son consideradas como fundamentales a la hora de que un egresado pretenda un puesto laboral. Identificándose el poco desarrollo de competencias blandas. Los futuros ingenieros no están exentos de esto. Los empleadores requieren, cada vez más, profesionales que posean, además de las competencias en ciencias duras, las competencias blandas desarrolladas para enfrentar a la sociedad actual. Vargas (2019), indicó: "Las habilidades blandas son definidas como características inherentes a cada persona que contribuye a su desempeño social" (p. 1). Según el Dr. Alex K. (citado en Vargas, 2019): En cualquier tipo de trabajo que se desarrolle, lo que realmente hará la diferencia no serán las competencias profesionales (o habilidades duras), sino lo que se conoce como "habilidades blandas". En las pruebas de entrada que se aplican para la Acreditación en la UNI y UNMSM, se les planteó a los estudiantes de ingeniería, algunas peguntas no cuantitativas, sino de opinión crítica, de soluciones a situaciones nunca vistas. Los resultados fueron preocupantes, de 100 estudiantes evaluados solo el 10\% contestó adecuadamente (Informes para Acreditación FIA -UNI, FIIS- UNI, FIEECS-UNI, FIQT- UNI, FIIS-UNMSM). En una encuesta de sondeo, realizada en diciembre de 2019, se les preguntó a 30 estudiantes de la UNI y 30 de San Marcos sobre características de aspectos como emociones, liderazgo, trabajo en equipo, realización de proyectos, redacción e idioma inglés, los resultados fueron, porcentaje nivel alto UNI 45\%, y en San Marcos 48\%. En una investigación extensiva a otra universidad de Lima, el investigador encontró los siguientes resultados. Asertividad, totalmente de acuerdo 30,3\%, Liderazgo 34,15\%, Conciencia y cultura 41\%. Lo que evidencia la falencia de estas habilidades en estudiantes pares. Al realizar una revisión de las mallas curriculares de las escuelas de ingeniería UNI y de San Marcos, encontramos que, de los cursos que favorecen el desarrollo de habilidades blandas, la UNI tiene en créditos 4,28\% y UNMSM 6,75\%, siendo así muy poco. (De un promedio de 220 créditos). Se observó cursos de oratoria, redacción y teatro en las mallas curriculares; en la UNI, solo de redacción; lo que podría marcar diferencias en habilidades blandas. Las universidades públicas en Lima están en proceso de adaptación y crecimiento, además se hace necesario desarrollar metodologías y técnicas de medición de desarrollo de habilidades blandas en los estudiantes y de los propios docentes que generen un efecto multiplicador. Dextre (2019), profesor principal de Gestión Tecnológica Empresarial de la Universidad Nacional de Ingeniería (UNI), añadió que actualmente no solo es necesario que un ingeniero sea analítico o que tenga gran conocimiento en temas específicos, sino que "además se requiere que sea flexible, integrador, comprometido, tolerante, asertivo, debe saber trabajar en equipo, tener habilidades comunicacionales, pasar de ser táctico a ser un estratega y ser un buen líder; es decir, saber hacer prospectiva. Esas habilidades blandas lo potencian para un ocupar un puesto de dirección en el trabajo" (Diario El Peruano). Por lo anterior, planteamos: 
- ¿Cuál es el nivel de las habilidades blandas que alcanzan los estudiantes de ingeniería de dos universidades públicas de Lima Metropolitana, 2020?

- ¿Qué grupo de estudiantes de ingeniería tiene mayor nivel?

- ¿Qué habilidades blandas tienen alto nivel en cada submuestra?

- ¿Qué habilidades blandas tienen más bajo nivel en cada submuestra?

Objetivo General. Determinar el nivel de habilidades blandas en estudiantes de Ingeniería de dos universidades públicas de Lima Metropolitana, 2020. Objetivo Específico. Comparar los niveles de habilidades blandas en estudiantes de ingeniería entre las universidades $A$ (UNMSM) y B (UNI), 2020. Especificar cuáles son las habilidades blandas que tienen nivel alto, en los referidos estudiantes. Conocer cuáles son las habilidades blandas menos desarrolladas en los estudiantes de ambas universidades. Sugerir, con base en los resultados, potenciar las habilidades blandas en los estudiantes de ambas universidades. Al realizar una revisión de las mallas curriculares de las escuelas de ingeniería UNI y de San Marcos, encontramos que, de los cursos que favorecen el desarrollo de habilidades blandas, UNI tiene en créditos (4,28\%) y UNMSM $(6,75 \%) \%$, siendo así muy poco. (De un promedio de 220 créditos). Se observó cursos de oratoria, redacción y teatro en las mallas de UNMSM; en UNI solo de redacción. Lo que podría marcar diferencias en habilidades blandas. Las universidades públicas en Lima están en proceso de adaptación y crecimiento, además se hace necesario desarrollar metodologías y técnicas de medición de desarrollo de habilidades blandas en los estudiantes y de los propios docentes que generen un efecto multiplicador.

\section{OBJETIVOS GENERALES}

Determinar el nivel de habilidades blandas en estudiantes de Ingeniería de dos universidades públicas de Lima Metropolitana, 2020.

\section{OBJETIVOS ESPECÍFICOS}

Comparar los niveles de habilidades blandas en estudiantes de ingeniería entre las universidades A (UNMSM) y B (UNI), 2020.

Especificar cuáles son las habilidades blandas que tienen nivel alto, en los referidos estudiantes.

Conocer cuáles son las habilidades blandas menos desarrolladas en los estudiantes de ambas universidades.

Sugerir, con base en los resultados, potenciar las habilidades blandas en los estudiantes de ambas universidades. 


\section{MARCO TEÓRICO}

\subsection{HABILIDADES BLANDAS}

Según Pérez (2009, citado en Vargas, 2019): "Las habilidades blandas son aquellos atributos que permiten actuar de manera efectiva. Confluyen una combinación de destrezas destinadas a tener una buena inter-relación; es decir, saber escuchar, dialogar, comunicarse, liderar, estimular, delegar, analizar, juzgar, negociar y arribar a acuerdos. Engloban un conjunto de aptitudes transversales e incluyen el pensamiento crítico, la ética y la posibilidad de adaptación al cambio".

De acuerdo con Heckman \& Kautz (2012, citado en Solís, 2019), las habilidades blandas no son ni inmutables ni innatas, sino que obedecen a un proceso de desarrollo a lo largo de toda la vida.

Según Laker, \& Powell (2011), los individuos que aprenden habilidades blandas, en comparación con las habilidades duras de aprendizaje, no están muy ligados a los fracasos.

Gibb (2014) define las habilidades blandas como "las habilidades intra e interpersonales esenciales para el desarrollo personal, la participación social y el éxito laboral (p.455), destacando que están fuertemente asociadas con el éxito personal y profesional.

Matus, O., \& Gutiérrez, A. (2017) indican que: las habilidades se pueden agrupar en cognitivas y no cognitivas o socioemocionales. Las cognitivas se vinculan directamente al coeficiente intelectual, a la esfera del conocimiento y saber académico. Estas también se pueden encontrar como habilidades duras. Las no cognitivas o socioemocionales que también se conocen como habilidades blandas pertenecen al área del comportamiento y son las que les permiten a las personas su relacionamiento con los demás.

La Corporación Industrial Minuto de Dios (2018) señaló que las habilidades más valoradas en la empresa son las siguientes. 
Tabla 1. Corporación Industrial Minuto de Dios (citado en Marrero, 2018)

\begin{tabular}{ll}
\hline Habilidades & $\%$ \\
\hline Solución de problemas & 62 \\
Pensamiento crítico & 60 \\
Adaptabilidad & 40 \\
Redacción & 44 \\
Comunicación oral & 38 \\
Análisis de datos & 36 \\
Trabajo en equipo & 36 \\
\hline
\end{tabular}

ABET (2020) señala las siguientes competencias o habilidades para los futuros ingenieros:

1. Estrategias de aprendizaje autónomo en los diferentes niveles y campos del conocimiento. 2. Utilizar los lenguajes lógico, formal, matemático, icónico, verbal y no verbal de acuerdo con su etapa de vida. 3. Dominar su lengua materna en forma oral y escrita con corrección. 4. Emplear pensamiento lógico, crítico, creativo y propositivo para analizar fenómenos naturales y sociales. 5. Utilizar un segundo idioma, preferentemente el inglés, con claridad y corrección para comunicarse en contextos cotidianos, académicos, profesionales y científicos. 6 . Elaborar propuestas académicas y profesionales inter, multi y transdisciplinarias de acuerdo con las mejores prácticas mundiales para fomentar $y$ consolidar el trabajo colaborativo. 7. Preocuparse por obtener un conocimiento y habilidad alternativa a su formación original. 8. Aprender con base en Proyectos que desarrollan aplicaciones prácticas de la ingeniería.

En este estudio, se tomará como referencia las habilidades que señala $A B E T$, por ser la acreditadora de las carreras de ingeniería que ha realizado un conocimiento crítico de los contenidos de estas carreras profesionales en el Perú; asimismo, es la que ha realizado las acreditaciones de las carreras en la UNI y UNMSM.

\section{REVISIÓN DE LA LITERATURA}

Sarmiento (2019), presentó su tesis Habilidades blandas necesarias para la formación del ingeniero de sistemas del siglo XXI. Universidad Piloto de Colombia. Bogotá.

El objetivo fue conocer cuáles son las habilidades que requieren las empresas en los ingenieros de sistemas. Estudio comparativo de las habilidades blandas clasificadas por el sector empresarial contra el plan de estudio del programa de Ingeniería de Sistemas de la universidad. Utilizó una encuesta en línea al sector empresarial, para comparar el plan de estudios del programa de Ingeniería de Sistemas de la universidad en estudio, con los requerimientos del empresariado. 
Los resultados indicaron que el empresariado opina que los recién egresados de Ingeniería de Sistemas no manejan las habilidades blandas necesarias para tener un buen desempeño en sus labores diarias. Además, más del $90 \%$ manifestó que el trabajo de estas destrezas se debe realizar a lo largo de todo el programa académico y no solo dejarlo para desarrollarlo en talleres o cursos complementarios.

Concluye que es necesario trabajar con los futuros ingenieros las habilidades como control emocional, buena comunicación, liderazgo y empatía para desempeñarse mejor en el mundo laboral.

Marrero y Amar (2018), en Habilidades blandas: necesarias para la formación integral del estudiante universitario. Revista científica Ecociencia, sostienen que el estudiante, al egresar de la educación superior e integrarse al ámbito laboral, se enfrenta a un cargo empresarial que exige un determinado conocimiento técnico o competencias profesionales que le permitan desempeñar de forma óptima su trabajo. Es decir, se trata de las competencias duras o Hard Skills. Para el desarrollo integral de una persona no basta solo con el conocimiento que pueda aprender, además de ello, es necesario un complemento de habilidades que le permitan comunicarse, tener autocontrol, confianza en sí mismo. En la actualidad, los empleadores demandan de profesionales competentes, tanto en habilidades duras, como en blandas, es decir, que combinen el saber hacer con el saber ser y saber convivir.

Palma, Miñán y Ríos (2011, citados en Maffioli y Giuliano, 2003) expresan que en estos tiempos:

El perfil de un buen ingeniero debe basarse en la capacidad y voluntad de aprender, el conocimiento sólido de las ciencias naturales básicas y el buen conocimiento de algún campo de la tecnología, además de los valores humanos generales. Por otra parte, tiene que estar preparado para el aprendizaje permanente y también debe poseer una buena comunicación y trabajo en equipo (...). (p. 2553).

\subsection{AUTORES NACIONALES}

Huamán y Quispe (2019). Nivel de competencias blandas en egresados de psicología de la ciudad de Arequipa, UNSA.

Indican que las habilidades blandas son necesarias para incursionar con éxito en el campo laboral. Objetivo determinar el nivel de competencias blandas en egresados de psicología de Arequipa. De enfoque cuantitativo, de tipo descriptivo comparativo, de diseño no experimental de corte transversal, la muestra se conformó con 256 egresados de psicología, de muestreo no probabilístico. 
Concluyeron que las competencias blandas de los egresados en estudio se encuentran en un nivel medio, sin embargo, y en nivel alto las competencias de conciencia emocional, logro, optimismo, desarrollo de personas y empatía, y nivel bajo, orientación al servicio y gestión de conflictos.

Vargas (2019). La existencia de habilidades blandas y su influencia en el desempeño laboral de los estudiantes de ingeniería industrial de la modalidad Gente que Trabaja de la Universidad Continental- Sede Arequipa. (Tesis de pregrado). Universidad Continental. Arequipa-Perú.

Tuvo como objetivo identificar las habilidades blandas en profesionales de Ing. Industrial. Objetivo: realizar un análisis de cómo influyen las habilidades blandas a nivel laboral y organizacional así también analizar cómo estas mejoran el desempeño en el mundo laboral. Tomaron en cuenta siete habilidades blandas relevantes en el ámbito laboral: Adaptación, Trabajo en equipo, Liderazgo, Toma de decisiones y manejo de conflictos, Comunicación efectiva, Autogestión y Autorregulación.

Claudet (2018). Habilidades Sociales y Rendimiento Académico en Estudiantes de la UCVCallao.

Esta investigación enfatizó la relación de la variable habilidades sociales que representa el nuevo concepto a desarrollar en el sector Educación y la variable rendimiento académico que es la medición respectiva del desarrollo del Currículum que asume hoy en día toda organización y todo individuo que convive en una sociedad.

Objetivo: Determinar la relación que existe entre las Habilidades Sociales y el Rendimiento Académico en los estudiantes, Universidad César Vallejo, Callao.

De tipo básico y de diseño no experimental, transversal y correlacional. Se utilizó la secuencia del método hipotético deductivo, con una población de 60 estudiantes. Se usó dos cuestionarios de 15 ítems cada uno, que fueron sometidos a juicio de expertos para la validez y fiabilidad, cuyos resultados fueron de 0,833 para el cuestionario de habilidades sociales y 0,817 para el cuestionario de rendimiento académico.

Se concluyó con índice de 0,513 que existe una relación inversa entre las habilidades sociales y el rendimiento académico en los estudiantes mencionados.

Ponce (2018) sostiene que, en la UNI, los estudiantes de la FIGMM - UNI no tienen mucha preparación para las entrevistas laborales, lo que dificulta su realización de prácticas, siendo esto una falencia que de no corregirse tendría una tendencia a acentuarse. 


\section{METOdOLOGÍA}

De enfoque cuantitativo, tipo descriptiva, de diseño no experimental de corte transversal. Según Bernal (2016), este tipo de enfoque nos permitirá emplear una serie de técnicas estadísticas, utilización de instrumentos cuantitativos de medición y de análisis que contribuirán a sustentar nuestros postulados. Utilizamos la técnica de la encuesta on line con un cuestionario de 22 ítems en tres dimensiones: Asertividad (11 ítems), Liderazgo (7 ítems) y Conciencia y cultura actual (4 ítems), basados en Claudet (2018) y los requisitos de ABET (2020).

Por ser descriptiva no se presenta hipótesis. (Hernández et al., 2018).

La población fueron todos los estudiantes de Ingeniería de UNMSM (11 000) y de UNI (10 000 ), a diciembre de 2019. La muestra fueron 224 , seleccionados por facultades de ambas universidades, por conveniencia.

Tabla 2. Muestra

\section{Muestra}

\begin{tabular}{lcc}
\hline \multicolumn{2}{l}{ Universidad } & Muestra \\
\hline UNMSM & (A) & 124 \\
UNI & (B) & 120 \\
\hline Total & 224 \\
\hline
\end{tabular}

Se envió correos a 400 estudiantes de la Universidad Nacional Mayor de San Marcos y a 300 estudiantes de la Universidad Nacional de Ingeniería, entre los meses de junio y julio del 2020.

\section{RESULTADOS}

Tabla 2. Habilidades Blandas en estudiantes de Ingeniería muestra 224 UNMSM (A), UNI (B).

\section{Nivel alto}

\begin{tabular}{lc}
\hline Universidad & $\%$ \\
\hline UNMSM & 29 \\
UNI & 23 \\
\hline
\end{tabular}

Nota: Consideramos las respuestas en totalmente de acuerdo. 
Figura 1: Comparativo en porcentaje de habilidades blandas, nivel alto UNI y UNMSM.

Habilidades Blandas Nivel Alto

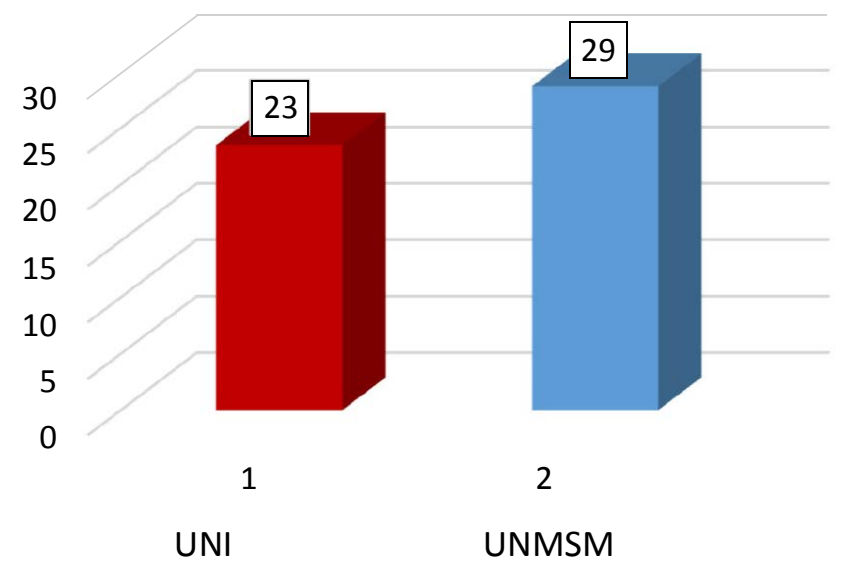

Al comparar el nivel alto en habilidades blandas de los estudiantes de Ingeniería de la Universidad A (UNMSM) se obtuvo un promedio de $29 \%$ y los estudiantes de la Universidad B (UNI), un porcentaje promedio de $23 \%$.

\section{Nivel medio}

Figura 2: Comparativo en porcentaje de habilidades blandas, nivel medio UNI y UNMSM.

\section{Habilidades Blandas Nivel Medio}

(\% Promedio)

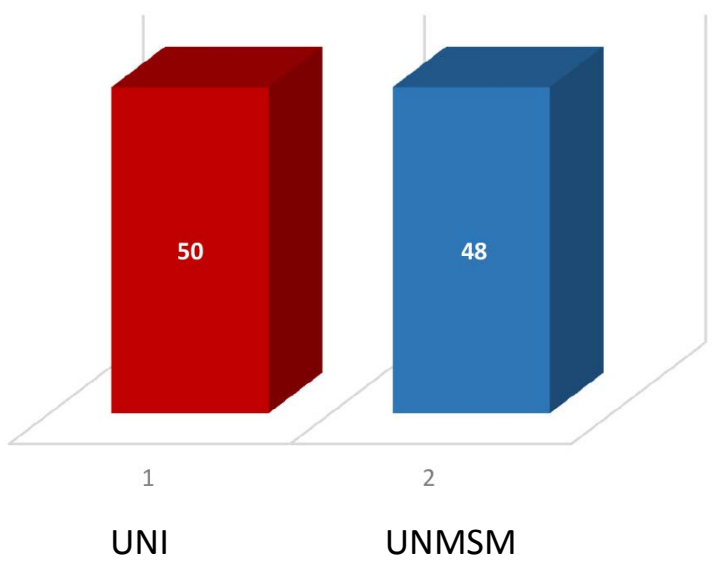

En cuanto a las habilidades blandas con resultado de nivel medio, los estudiantes de Ingeniería de la Universidad B (UNI) obtuvieron un porcentaje promedio de $50 \%$, mayor en $2 \%$, de los estudiantes de la Universidad A (UNMSM) obtuvo $48 \%$. 
Figura 3: Comparativo en porcentaje de habilidades blandas, nivel regular UNI y UNMSM

Habilidades Blandas Nivel Regular (\%)

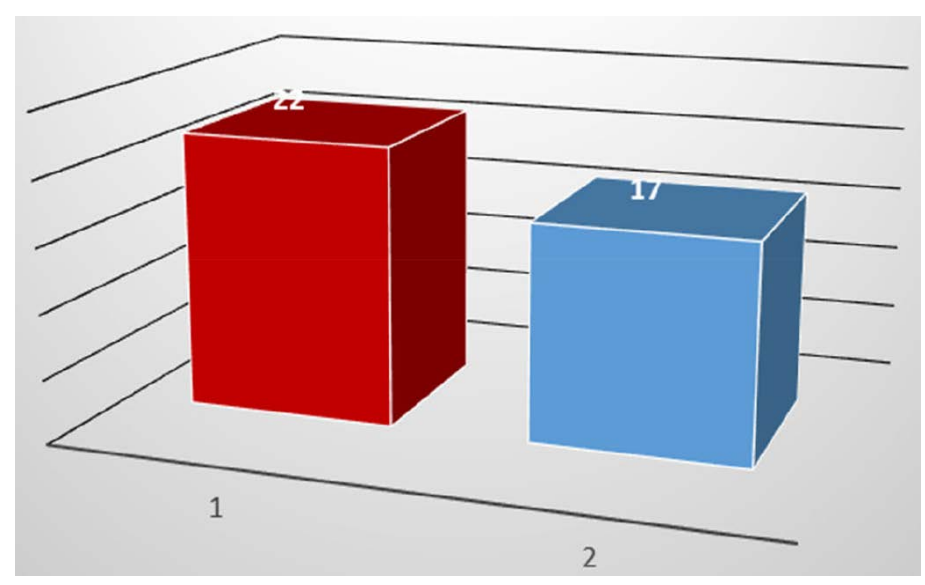

UNI

UNMSM

En el resultado de nivel regular de porcentajes de los estudiantes de Ingeniería de ambos, los de la Universidad B (UNI), se autocalifican como de nivel regular en promedio $22 \%$ y los de la universidad A (UNMSM), en promedio $17 \%$, con una diferencia de $5 \%$.

Tabla 3. Habilidades por universidades y dimensiones

Dimensiones

\begin{tabular}{llll}
\hline Universidad & $\begin{array}{l}\text { Asertividad } \\
\text { (Aceptable) }\end{array}$ & $\begin{array}{l}\text { Liderazgo } \\
\text { (Aceptable) }\end{array}$ & $\begin{array}{l}\text { Conciencia y Cultura actual. } \\
\text { (Aceptable) }\end{array}$ \\
\hline UNMSM(A) & $49 \%$ & $38 \%$ & $43 \%$ \\
UNI(B) & $47 \%$ & $42 \%$ & $42 \%$ \\
\hline
\end{tabular}

Fuente: Encuestas

De la tabla 3, los resultados indican un porcentaje aceptable, pero no suficiente para las demandas de los empleadores. Se observa diferencias mínimas en porcentajes de nivel aceptable sin evidenciar una preponderancia.

\section{DISCUSIÓN DE RESULTADOS}

En cuanto al objetivo general de determinar los niveles de habilidades blandas en los estudiantes de ambas universidades y realizar la comparación, se obtuvo que los estudiantes de Ingeniería de la universidad en términos generales en habilidades blandas desarrolladas, tienen bajos porcentajes en niveles aceptables, Universidad A, 29\% y 
Universidad B, 23\%, lo cual es consistente con lo hallado por Claudet (2018) en estudiantes de la UCV-Callao. Lo mismo que Ponce (2018), quien encontró ausencia de Liderazgo y otras habilidades blandas en los estudiantes de la FIGMYM- UNI. Concluyendo que es necesario trabajar con los futuros ingenieros las habilidades como control emocional, buena comunicación liderazgo y empatía para desempeñarse mejor en el mundo laboral. Situación que se presenta también en los estudiantes de ingeniería del presente estudio.

Los estudiantes UNI presentaron una ligera diferencia de $2 \%$ en la apreciación sobre habilidades blandas, lo cual indicaría que en su autoevaluación tomada por medio del cuestionario, la opinión de ambos es casi similar.

De las respuestas en nivel totalmente de acuerdo, de acuerdo y medianamente de acuerdo, se aprecia que los estudiantes de la Universidad $B$, se autoevalúan que sus habilidades blandas tienden de nivel medio a regular, mientras que los de la Universidad A, con un mayor porcentaje en nivel alto.

Objetivo secundario 1, establecer cuáles son las habilidades que tienen mayor desarrollo en los estudiantes de ambas universidades. Los estudiantes de la universidad A tienen mayor porcentaje en habilidades blandas en la respuesta totalmente de acuerdo, con una diferencia de $6 \%$. Sin embargo, aún sigue siendo preocupante el nivel ya que estarían presentando poca preparación en competencias como empatía, trabajo en equipo, control de emociones, conciencia y actualidad que contiene metacognición y aprendizaje basado en proyectos, requisito de acreditación de ABET. Coincide con la investigación de Huamán y Quispe (2019), quienes concluyeron que el nivel de competencias blandas de los egresados de psicología se encuentra en un nivel medio.

Objetivo secundario 2, Conocer cuáles son las habilidades blandas menos desarrolladas en los estudiantes de ambas universidades. Se encontró en las subdimensiones como aceptar las opiniones de sus compañeros, respuestas de $30 \%$ en medianamente de acuerdo (Universidad A) y 32\% (Universidad B). Dominio del idioma inglés en desacuerdo, 23\% Universidad A y $35 \%$, universidad B.

Objetivo secundario 3, en relación con el aporte esperado de la presente investigación, indicamos: El desarrollo de habilidades blandas en estudiantes de educación superior tiene que ser trabajado desde el entendimiento de programas y estrategias que potencien dichas habilidades. Las habilidades blandas se desarrollan mediante el modelamiento y el aprendizaje activo, donde por medio de Planes de estudio (Mallas) de estructura transversal se insertan las habilidades dentro de los diversos cursos ciclo por ciclo. Tenemos, el aprendizaje basado en proyectos (ABP2) y aprendizaje basado en problemas $(A B P)$. La empresa acreditadora ABET, precisamente exige el desarrollo de Proyectos. Olmedo et al (2016), indican: El Aprendizaje Basado en Problemas (ABP) es una 
metodología docente centrada en el estudiante siendo este el protagonista de su propio aprendizaje, facilita la adquisición de conocimientos y ayuda al desarrollo de competencias profesionales específicas y genéricas. Lo que concuerda con Sarmiento (2018), quien concluyó que es necesario trabajar con los futuros ingenieros las habilidades como control emocional, buena comunicación liderazgo y empatía para que se desempeñen mejor en el mundo laboral. Esto es consistente con Dextre (2020), quien señala que los ingenieros de éxito deben desarrollar no solo conocimientos técnico-científicos, sino también habilidades como empatía, asertividad, trabajo en equipo que los hace más competitivos.

\section{CONCLUSIONES}

- Respecto al objetivo general, se halló que los niveles aceptables alcanzados fueron: Dimensión asertividad UNI (45\%), UNMSM (52\%), Liderazgo, UNI (46\%), UNMSM (43\%), Conciencia y actualidad, UNI (46\%), UNMSM (48\%). Por lo que no existen diferencias preponderantes en habilidades blandas entre los estudiantes de Ingeniería de la Universidad A y la Universidad B.

- Existe una diferencia mínima entre la valoración de los estudiantes de la Universidad A y la Universidad B en acercamiento interpersonal de sus pares. Unos (A) parecen tener mayor apego al aspecto colaborador y cooperativo. Los estudiantes de la Universidad B obtuvieron porcentaje de $25 \%$ en desarrollo de Proyectos, en cambio los de la Universidad A, alcanzó un 42\%, en totalmente de acuerdo.

- En la dimensión conciencia y cultura actual, en las dos muestras se notó que el 90\% son conscientes de entablar un proyecto de vida con responsabilidad hacia el medio y estar en permanente capacitación que los haga más competitivos en el mercado laboral.

- En cuanto al objetivo 3, se encontró que las habilidades blandas con más porcentajes fueron en UNI Asertividad (45\%) y UNMSM (48\%).

- Se halló que las habilidades blandas menos desarrolladas fueron en UNI dominio del inglés (30\%), medianamente y en UNMS (28\%), en comunicación UNI (37\%) y UNMSM (35\%).

- De acuerdo con las pretensiones de la investigación, para la docencia universitaria serviría como base de evaluación de cada una de sus clases y así reajustar los sílabos para mejorar o desarrollar las habilidades blandas desde todos los campos que se observe la carrera. 
Para los estudiantes se identificó el nivel de habilidades blandas a la fecha de corte de la investigación y luego hacerles conocer las falencias que los hacen menos elegibles a la hora de incursionar en el mercado laboral, tomen conciencia y cambien su visión.

\section{RECOMENDACIONES}

Se recomienda que las autoridades de ambas universidades implanten cursos que favorezcan el desarrollo de habilidades blandas como oratoria, coaching, redacción con reglas para una investigación, teatro, análisis y pensamiento crítico, inglés intensivo y proyecto de fin de ciclo con carga obligatoria.

Se recomienda que los docentes de ambas universidades tomen conciencia de que no solo se diserta sobre la materia de su conocimiento sino también promover el desarrollo de habilidades blandas con trabajos en equipo, presentación de proyectos, uso del idioma inglés, la metodología y redacción de trabajos con normas APA y otras, así como la presentación y exposición de los temas.

Esto se viene impulsando con la metodología de enseñanza mediante los trabajos en línea, las plataformas de clase y las participaciones on line de los estudiantes.

- Realizar seminarios de Habilidades Blandas dirigidos a los estudiantes como fin de ciclo y que participen en los eventos programados (Ferias de Proyectos) con temas inéditos en grupo, exposiciones en inglés y escribir un artículo como parte de las prácticas preprofesionales que sean considerados en su récord de créditos de su carrera.

- Se sugiere, realizar otras investigaciones acerca de las debilidades o falencias de los estudiantes en cuanto a la adquisición de habilidades blandas como la comunicación, la participación más comprometida en los trabajos, el liderazgo y el dominio del idioma inglés. 


\section{REFERENCIAS}

Alles M. A. (2018). Desarrollo del talento humano Buenos Aires: Granica, 2005. Recuperado de https:// www.academia.edu/33494469/ Desarrollo del Talento Humano Basado en Competencias de Alles Martha

Barreto, J. P. y Izquierdo, C. A. (2017). La importancia del coaching en el desarrollo de las habilidades blandas del personal de la empresa MARCIMEX en la ciudad de Trujillo año 2016 (Tesis de pregrado). Universidad Privada Antenor Orrego, Trujillo. Recuperado de http://repositorio.upao.edu.pe/handle/ $\underline{20.500 .12759 / 3460}$

Cabrero, B. (2018). Las habilidades socioemocionales, no cognitivas o "blandas": aproximaciones a su evaluación. Revista Digital Universitaria, 19(6). Recuperado de http://doi.org/10.22201/ codeic.16076079e.2018.v19n6.a5

Claude, M (2018). Habilidades Sociales y Rendimiento Académico en Estudiantes de la Universidad Cesar Vallejo, Sede Callao 2018. Recuperado de https://hdl.handle.net/20.500.12692/17440

Corporación Industrial Minuto de Dios. (2018). Habilidades duras y habilidades blandas, Recuperado de https://mdc.org.co/blog-formacion-habilidades-duras-habilidades-blandas/

Ghenadenik (2017). El rol del docente en la universidad. Reflexión académica en diseño y comunicación Nro XXX. Recuperado de https://fido.palermo.edu/servicios dyc/publicacionesdc/archivos/636 libro.pdf

Huamán y Quispe (2019). Nivel de competencias blandas en egresados de psicología de la ciudad de Arequipa. Tesis de pregrado. UNAS.

El Comercio (2019). "Habilidades blandas: así influyen en tu carrera" Recuperado de: https://elcomercio.pe/ suplementos/comercial/carreras-gente-trabaja/que-son-habilidades-blandas-y-como-influyen-tucarrera-1003756

Fernández, B. (2007). Competencias laborales y de empleabilidad en la educación vocacional. Calidad en la Educación, (27), 36-51. doi:https://doi.org/10.31619/caledu.n27.217

Gestión (2019). Las 10 habilidades blandas que demandará el mercado laboral en el futuro. Recuperado de https://gestion.pe/economia/management-empleo/10-habilidades-blandas-demandara-mercadolaboral-futuro-nnda-nnlt-261487 .uned.es/fez/eserv/bibliuned:EducacionXXI-2007numero10-824/Documento.pdf

Gómez-Gamero, M. E. (2019). Las habilidades blandas competencias para el nuevo milenio. Divulgare Boletín Científico de la Escuela Superior de Actopan, 6(11). Recuperado de https://doi.org/10.29057/ esa.v6i11.3760

Hernández, R., Fernández, C., y Baptista, P. (2014). Metodología de la Investigación. D.F., México: McGrawHill. 
Hernández, G. (2019). Formación dual, oportunidad para mejorar la empleabilidad. El economista. Recuperado de https://factorcapitalhumano.com/mundo-del-trabajo/formacion-dual-oportunidadpara-mejorar-la-empleabilidad/2019/02/

Iparraguirre, L. (2019). Perú: egresados demoran hasta seis meses para encontrar empleo. Agencia Peruana de Noticias, Andina. Recuperado de https://andina.pe/agencia/noticia-egresados-demoran-tres-aseis-meses-para-encontrar-trabajo-742180.aspx

Maya, M. T. y Serrano, B. S. (2016). Desarrollo de soft skills una alternativa a la escasez de talento humano. INNOVA Research Journal, 1(12), 59-76. Recuperado de http://www.journaluidegye.com/magazine/ index.php/innova/article/view/81/142

Millalén, F. V. (2016). Inclusión de habilidades blandas en el currículo de la educación superior: Clave para el desarrollo de capital humano avanzado. Revista Akadèmeia, 15(1) ,53-73. Recuperado de http:// revistas.ugm.cl/index.php/rakad/article/view/137/129

Pérez, Wilfredo. 2017. ¿Qué son las habilidades blandas? Gestiopolis. [En línea] 22 de 09 de 2017. [Citado el: 14 de 12 de 2018.] Disponible en https://www.gestiopolis.com/las-habilidades-blandas/.

Ponce, Lily (2018). Estilo de liderazgo en estudiantes del quinto año de la Facultad de Ingeniería Geológica, Minera y Metalúrgica de la Universidad Nacional de Ingeniería. Tesis de posgrado.UPCH- Lima. Perú.

DR. K. ALEX. (2016), libro: "Soft Skills, Know Yourself and Know the World". (Habilidades blandas, conócete a Ti mismo, conoce el mundo)

Santos, C. E. O. (2017). Desarrollo de habilidades blandas desde edades tempranas. Recuperado de http:// www.ecotec.edu.ec/content/uploads/2017/09/investigación/libros/desarrollo-habilidades.pdf

Sánchez, O. M., Amar, R. M., y Triadú, J. X. (2018,20 de noviembre). Habilidades blandas: necesarias para la formación integral del estudiante universitario. Revista Científica ECOCIENCIA, edición especial, 1-18. Recuperado de https://doi.org/10.21855/ecociencia.50.144

Silva, C. J. (2016). Brecha porcentual entre las habilidades blandas de los estudiantes de relaciones industriales y las requeridas por empresas de Yanahuara-Arequipa 2016 (Tesis de pregrado). Universidad Nacional de San Agustín, Arequipa, Perú.

Suárez, J. R. (2018). Conocimientos y habilidades necesarios en la era digital: sus cuatro dimensiones. Think Big. Recuperado de https://empresas.blogthinkbig.com/conocimientos-y-habilidades-necesarios-enla-era-digital/

Tobón, S. (2008). La formación basada en competencias en la educación superior: el enfoque complejo. Universidad Autónoma de Guadalajara México: Curso IGLU. Recuperado en: http:// cmapspublic3.inmc.us/rid=1LVT9TXFX-1VKCOTM-16YT/ Formaci\%C3\%B3n\%20basada\%20en\%20competencias\%20(Sergio\%20Tob\%C3\%B3n).pdf

Torres, E. L. (2018). Causas de la falta de aprovechamiento de las habilidades blandas en la empresa AEGOCEP Perú S.A.C. (Tesis pregrado). Universidad Nacional de San Agustín, Arequipa, Perú. Recuperado de http://repositorio.unsa.edu.pe/bitstream/handle/UNSA/7259/ Rltorael.pdf?sequence $=1 \&$ isAllowed $=\mathbf{y}$ 
Vicerrectorado Académico de la PUCP. (2019). Competencias genéricas en la PUCP. Recuperado de http:// vicerrectorado.pucp.edu.pe/academico/noticias/competencias-genericas-la-pucp/

Wheeler, R. 2016. Soft Skills - The Importance of Cultivating Emotional Intelligence. Recuperado de https:// scholarship.law.bu.edu/faculty scholarship/130

Los artículos publicados por IECOS pueden ser compartidos a través de la licencia Creative Commons: CC BY 4.0 Perú. Permisos lejos de este alcance pueden ser consultados a través del correo revistas@uni.edu.pe.

(c) (i) 